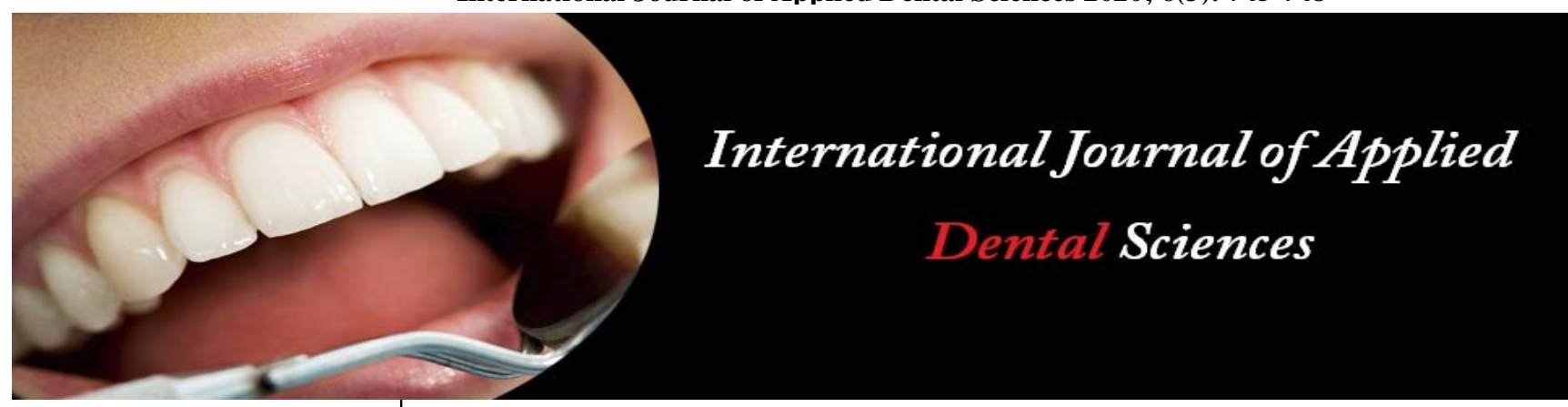

ISSN Print: 2394-7489 ISSN Online: 2394-7497 IJADS 2020; 6(3): 745-748 (C) 2020 IJADS www.oraljournal.com Received: 20-05-2020 Accepted: 23-06-2020

Dr. Abhishek Sharma MDS Prosthodontics, Crown Bridge and Implantology. Himachal Pradesh, India MDS, Senior lecturer, Department of Prosthodontics, Crown Bridge and Implantology, Bhojia Dental College and Hospital, Bhud, Baddi,

Himachal Pradesh, India

Dr. Sonali Sharma

MDS $2^{\text {nd }}$ year student, Department of Prosthodontics, Crown Bridge and Implantology, Bhojia Dental College and Hospital, Bhud, Baddi, Himachal Pradesh, India

Dr. Shivangi Sahi BDS, Government Dental College, Shimla, India

Dr. Vishal Thakur BDS, Dental Surgeon at Ekdant Dental Clinic, Himachal Pradesh, India

Priyanka Jamwal BDS final Year student, Himachal Dental College, Sundernagar, Himachal Pradesh, India
Dr. Ramandeep Singh

\section{A review on "Factors affecting osseointegration in dental implants”}

\author{
Dr. Abhishek Sharma, Dr. Ramandeep Singh, Dr. Sonali Sharma, Dr. \\ Shivangi Sahi, Dr. Vishal Thakur and Priyanka Jamwal
}

DOI: https://doi.org/10.22271/oral.2020.v6.i3k.1031

\section{Abstract}

Osseointegration was discovered in 1962 and coined as a term in 1977. Original definitions implied direct contact between foreign materials and bone without any interposed soft tissue layers. Today, osseointegration is regarded to be a foreign body response to separate foreign elements from bone. A new definition of the term is suggested in this paper; "Osseointegration is a foreign body reaction where interfacial bone is formed as a defense reaction to shield off the implant from the tissues".

Keywords: "osseointegration, dental implants", foreign body reaction

\section{Introduction}

Per-Ingvar Brånemark of Sweden. started placing experimental implants in rabbits and with time in $\operatorname{dog}$ s and found them impossible to remove without fracturing the bone ${ }^{[1]}$.

Brånemark selected commercially pure (c. p.) titanium as material for his implant to be and he then constructed a threaded implant that was hollow with glass rods glued inside. In fact, two glass rods were separated by a space of about 100 micrometers. When this implant was screwed home through the tibial bone of a rabbit, he was able to decide whether the space would be placed in the marrow zone or in the cortical bone ${ }^{[2,3]}$. He wants to study whether the blood vessels get adhered to the implant, after placement of implant in the surgical site and he was proven right, not only the blood vessels grew near or around the implant but, the bone tissue also grew up around the implant, when the implants are placed properly, precisely at a good position with good surgical site ${ }^{[4]}$. Brånemark's serendipity based observation that his implant chambers remained most difficult to remove from the bone, immediately made him conclude that the implants were anchored in bone tissue ${ }^{[5,6]}$. This was the concepts of those days that saw a direct bone anchorage of any metallic specimens as impossible.

Osseointegration can be defined as:-

- 1969- "As a direct contact between the bone and metallic implants without interposed soft tissues layers".

- 1977- "Direct structural and functional connection between ordered, living bone and the surface of a load carrying implant".

- According to GPT-8 "the apparent direct attachment or connection of osseous tissue to an inert, alloplastic material without intervening connective tissue"

- Meffert et al (1987) Adaptive Osseointegration: Osseous tissue approximating the surface of the implant without apparent soft tissue interface at light microscopic level.

\section{Factors Effecting Osseointegration ${ }^{[7,8,9]}$}

Six different factors known to be important for the establishment of a reliable, long-term osseous anchorage of an implanted device

Corresponding Author: Dr. Abhishek Sharma MDS Prosthodontics, Crown Bridge and Implantology. Himachal Pradesh, India
- Implant biocompatibility

- Design characteristics

- Surface characteristics

- State of the host bed 
- $\quad$ Surgical technique and

- Loading conditions

\section{Implant Biocompatibility ${ }^{[7,8,9]}$}

Response of bone to different implant material is the principal factor on which an implant material is selected as suitable or unsuitable for osseointegration

- Chemical interaction determined-properties of surface oxide Commercially pure (c.p.) Titanium and Titanium alloy (Ti -6AL-4V) and it shows

- Documented long term function

- Covered with adherent, self- reparing oxide layer

- Excellent resistance to corrosion-high dielectric constant

- Load bearing capacity

- The mechanical properties of Titanium alloy are superior to C.P-Titanium

\section{Other metals}

- Niobium, tantalum

- Cobalt chrome molybdenum alloys

- Stainless steels

- Ceramics-calcium phosphate hydroxyapatite (HA) and various types of aluminium oxides

Implant Design (Macrostructure) ${ }^{[10,11]}$

Threaded or screw design implants

- Demonstrated to function for decades without clinical problems.

- Provide more functional area for stress distribution than the cylindrical implants.

- Minimal- $<0.2 \mathrm{~mm} /$ year bone loss

Cylindrical implants ${ }^{[7,8,9]}$

Press fit root form implants depend on coating or surface condition to provide microscopic retention and bonding to the bone

\section{Combination root forms}

- Macroscopic features of cylinder and screw root forms

\section{The design of the threads}

The dental implant applications dictate the need for a thread shape optimized for long term function, load transmission under occlusal, intrusive and shear loading

Functional surface area per unit length of implant may be modified by the three thread geometry parameters

- Thread shape

- Thread pitch

- Thread depth

- $\quad$ Square thread

Optimized surface area for intrusive and compressive loads Shear force 10 times lesser than Vshape

- Decreased thread pitch increases the functional surface area surface area

- The greater the thread depth the greater the surface area of the implant, if all factors are equal

A wider diameter, more threads, deeper threads and surface structure that increase the initial bone contact percentage are of great benefit. Alterations in these are suggested according to Bone density.
Grooves on implants ${ }^{[10,11]}$.

Grooves on the threads of all implants and on the collars, where ever appropriate.

- Increase surface area

- Increase area for bone-to-implant contact

Implant Surface ${ }^{[7,8,9]}$ (Microstructure, Surface Topography) "The extent of bone implant interface is positively correlated with an increasing roughness of the implant surface"

\section{Roughened surface}

- Greater bone to implant contact at histological level

- Micro irregularities-cellular adhesion.

- High surface energy-improved cellular attachment.

Some Methods To Increase The Surface Topography:-

- Electroploishing

- Sand blasting

- Sand blasting and acid attack

- Titanium plasma spraying

The titanium with highest bone to implant contact was created with large grit balating and acid attack.

This surface gave similar bone to implant contact as did implants coated with hydroxy apatite. Surface coatings

Two materials plasma sprayed onto implant body

- Titanium

- Hydroxyapatite

\section{Advantages of TPS}

- Increased surface area

- Increased roughness for initial stability

- Stronger bone to implant interface

\section{Advantages of Hydroxyapatite on TPS}

- Faster healing bone interface

- Stronger interface than TPS

- Less corrosion of metal

\section{State of the Host}

Ideal host bed should be

- Healthy and with an adequate bone stock

- Having adequate bine height

- Having adequate

- Having adequate bone height

- Having adequate bone density

Undesirable host bed states for implantation

- Ridge height resorption

- Osteoporosis

- $10-15 \%$ poorer clinical results must be anticipated after a therapeutic dose of irradiation - vascular damage, at least in part.

- $\quad$ Such clinical states may constitute an indication for ridge augmentation with bone grafts.

- As stated by Branemark et al and Misch, the bones with D1 and D2 bone densities shows good initial stability and better osseointegration.

\section{Surgical Considerations}

The main aim of the careful surgical preparation of the implant bed is to promote regenerative type of the bone healing rather than reparative type of the bone healing. 
- The critical time/ temperature-bone tissue necrosis- $47^{\circ}$ for one minute.

Recommendations by Erickson R.A.

- Slow speed

- Graded series

- Adequate cooling

- Overheating avoided

- Bone cutting speed of less than $2000 \mathrm{rpm}$

- Tapping at a speed of $15 \mathrm{rpm}$ with irrigation

- Using sharp drills

The surgical preparation sequences as well as the instruments depend upon the quality of the bone.

Number of drills used to prepare implant osteotomy corresponds to bone density

- D1 uses six drills and D3 uses four Drills

- D4 uses osteotomes to compact fine trabecular bone.

- Bone taps are used for D1 and D2 bone.

- Countersink drill is optional in D3 bone

\section{Power used at implant insertion}

- Too strong a hand will use in bone tension and a resorption response will be stimulated.

- Holding power of the implant will fall to dangerous levels after a strong insertion torque.

- A moderate power at the screwing home of an implant is therefore recommended.

- The optimal torque threshold - $35 \mathrm{~N} / \mathrm{cm}$.

- Implant should gently engage the bone in order to avoid too much pressure at the bone interface which could jeopardize healing

\section{Surgical fit of the fixture}

- The accurate fit consists of more surface contact, less dead space and thus better healing.

\section{Loading Conditions}

\section{Progressive or two stage loading}

- Immediate or one stage or non submerged loading

- Progressive or two stage loading

Branemark et al to accomplish osseointegration considered the following prerequisites

- Countersinking the implant below the crestal bone

- Obtaining and maintaining a soft tissue covering over the implant for 3 to 6 months

- Maintaining a non-loaded implant environment for 3 to 6 months

Elements of progressive loading

- Time interval

- Diet

- Occlusal material

- Occlusal contacts

- Prosthesis design

\section{Time}

The healing time between the initial and second stage surgeries for

- $\mathrm{D} 1$ and D2 is similar-3 to 6 months

- $\quad$ D3 and D4-5 to 6 months

- 3 month loading delay in the mandible and a 4-6 month delay in the healthy maxillary bone - more cancellous in character.

Diet

The dentist controls diet of the patient to prevent overloading.

- Initial healing phase- avoid chewing in the area.

- Initial transitional prosthesis to final prosthesis-soft diet

- After evaluation of final prosthesis function, occlusion and cementation-normal diet.

Occlusion

- Initial healing-no occlusal contacts

- First transitional prosthesis left out of occlusion in partially edentulous patients

- No occlusal contacts on cantilevers

- Final restoration - implant protective occlusion concepts.

The salient features of Branemark and his team's work

- About more than 50 designs of Ti screws (implants) were tested and used.

- The surgical protocol followed was: two stage surgery, which was proved beneficial.

- Minimal trauma during the surgery results in bone regeneration rather than bone repair at the implant site.

- Non-contaminated implants (sterile and clean implants) prove good integration.

- Prosthesis and abutments were screw attached for more technical flexibility.

- There were more mechanical failures at the interface rather than biological failures.

Methods of Evaluation of Osseointegration

- Stability is a requisite characteristic of osseointegration.

- When an implant is placed surgically, initial stability is a function of the bone quality, implant deign and surgical technique.

- Implant placed in the dense cortical bone should have higher initial stability than in a weak cancellous bone

- During the osseointegration healing and maturation process, the initial stability changes with increases in bone- to-implant contact and osseous remodeling.

- It is unknown however what precisely constitutes "adequate stability" to warrant proceeding with restoration

Rigid fixation: Absence of observed clinical mobility.

- Two terms osseointegration and rigid fixation are interchangeably used.

- First clinical criterion to be evaluated

- A healthy implant moves less than 73microns - appears as zero clinical mobility.

Invasive Methods

- Histological sections (10 microns sections)

- Histomorphometric-To know the percentage of bone contact

- Transmission electron microscopy

- By using Torque gauges

Non-Invasive Methods

- Percussion test

- Tapping with a metallic instruments 
The fixture produces ringing sound-osseointegrated.

Dull sound-fibrous integration.

- Radiographs

- Perio-test

- Dynamic modal testing

- Resonance frequency analysis

- Impulse testing

- Implatest

The Success Criteria (Alberktsson et al)

- The individual unattached implant should be immobile when tested clinically.

- The radiographic evaluation should not show any evidence of radiolucency.

- The vertical bone loss around the fixtures should be less than $0.2 \mathrm{~mm}$ per year after first year of implant loading.

- The implant should not show any signs of pain, infection, neuropathies, parasthesia, violation of mandible canals and sinus drainage.

- The success rate of $85 \%$ at the end of 5 year and $80 \%$ at the end of 10years

\section{Conclusion}

- As the concept of osseointegration has developed and spread globally, it has had a dramatic impact on the practices of dentistry.

- In implant dentistry, an undisturbed healing period is always required to ensure osseointegration.

- A modified protocol with early or immediate loading has been tested to satisfy the demand of more rapid treatment and to reduce discomfort of wearing removable appliances during the healing period.

- Provided that the implant has primary stability, studies have shown that the survival of loaded implants can be analogous to the unloaded protocol.

\section{References}

1. Osborn JF, Newesly H. Dynamic aspects of the implantbone interface. In: Dental Implants, Materials and Systems Heimke G, editor. Hanser Verlag, München, Wien 1980.

2. Albrektsson $\mathrm{T}$. The response of bone to titanium implants. CRC critical reviews in biocompatibility 1985;1:53-84.

3. Albrektsson T, Brånemark PI, Hansson HA, Kasemo B, Larsson K, Lundström I, et al. The interface zone of inorganic implants in bone. Ann Biomed Eng. 1983;11:141.

4. Sul YT. On the bone response to oxidized titanium implants: The role of microporous structure and chemical composition of the surface oxide in enhanced osseointegration 2002, 1-184.

5. Donath K, Laas M, Günzl H. The histopathology of different foreign body reactions in oral soft tissue and bone tissue. Virchows Arch A Pathol Anat Histopatol. 1991;420:131-137.

6. Donath K. Pathogenesis of bony pocket formation around dental implants. J Dent Assoc S Afr. 1992;47:204-208.

7. Suska F, Esposito M, Gretzer C, Källtorp M, Tengvall P, Thomsen P. IL- $1 \alpha$, IL- $1 \beta$ and TNF- $\alpha$ secretion during in vivo/ex vivo cellular interactions with titanium and copper. Biomaterials 2003;24:461- 468.

8. Suska F, Emanuelsson L, Johansson A, Tengvall P, Thomsen P. Fibrous capsule formation around titanium and copper. J Biomed Mater Res A 2008;85:888-896.

9. Bos I. Gewebereaktionum gelockerte Hüftgelenkendoprothesen. Der Orthopäde 2001;30:881-889.

10. Christiansen RJ. Metal release from implants and its effect on the immune system 2016, 199.

11. Thiele A, Bilkenroth U, Bloching M, Knipping St. Fremderkörperreaktion nach implantation eines biocompatible en OsteosyntheseSystems. HNO 2008;56:545-548.

12. Anderson J, Rodriguez A, Chang D. Foreign body reaction to biomaterials. Semin Immunol 2008;20:86100 . 